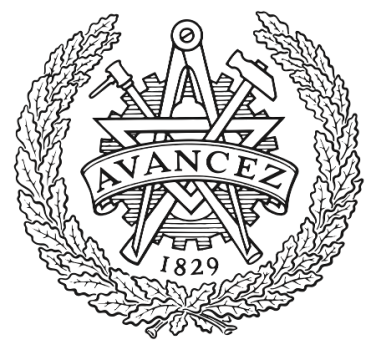

CHALMERS

UNIVERSITY OF TECHNOLOGY

\title{
All Doors Lead to the Kitchen - Sustainability and Wellbeing Challenges in a Shared Centrepiece of Living
}

Downloaded from: https://research.chalmers.se, 2023-04-26 10:32 UTC

Citation for the original published paper (version of record):

Andersson, S., Rahe, U. (2019). All Doors Lead to the Kitchen - Sustainability and Wellbeing Challenges in a Shared Centrepiece of Living. Advances in Intelligent Systems and Computing, 876: 111-116. http://dx.doi.org/10.1007/978-3-030-02053-8_18

N.B. When citing this work, cite the original published paper. 


\title{
All Doors Lead to the Kitchen - Sustainability and Wellbeing Challenges in a Shared Centrepiece of Living
}

\author{
Sofie Andersson ${ }^{(\bowtie)}$ and Ulrike Rahe \\ Department of Industrial and Materials Science, Division of Design \& Human \\ Factors, Chalmers University of Technology, 41296 Gothenburg, Sweden \\ \{sofiean, ulrike.rahe\}@chalmers.se
}

\begin{abstract}
The kitchen figures a central place in the home where a significant share of a household's resource consumption takes place. Sharing the kitchen between multiple households has potential to bring positive sustainability effects due to more efficient use of both material resources and energy. The concept of shared kitchens has, however, thus far had a limited diffusion. This paper explores the potential of shared kitchens as a future sustainable living environment by studying user experiences from a Living Lab setting. It builds the base for an overarching larger European collaboration on how future shared kitchens should be designed in order to support everyday practices while optimising the conditions for achieving positive impact on both sustainability and wellbeing. Findings are presented from five focus areas concerning different use contexts: (1) accessing, (2) cooking, (3) living and socialising, (4) storing, and (5) cleaning.
\end{abstract}

Keywords: Kitchen $\cdot$ Sharing $\cdot$ Sustainability $\cdot$ Wellbeing $\cdot$ Living lab

\section{Introduction}

The average household size in the European Union is decreasing, with single households accounting for one third of all households, thereby being the most common group as well as the one that has increased the most during the last decade [1]. Smaller households are in general less resource-efficient than larger households where space, energy, furnishings and transportation are shared between several household members $[2,3]$. Although energy efficiency of buildings and appliances has improved significantly over the last 30 years, a growing demand for living space and appliances has been found to counterbalance energy efficiency improvements from technological developments [4].

Despite increasing incomes and consumption in Western countries, wellbeing levels have been found to remain static and even decline, which is a paradox that indicates potential to reduce consumption without compromising levels of wellbeing [5]. An opportunity to step away from the individualistic and materialistic path towards wellbeing is offered by the concept of sharing. Sharing is an ancient, fundamental human behaviour [6] that, despite offering resource saving potentials [7] as well as social and 
economic benefits, has thus far had a limited diffusion $[8,9]$. It is therefore interesting to explore ways to create sustainable living solutions that could appeal to a wider group of people, allowing for compromises between private and shared spaces [10].

The kitchen figures a central place in the home that is becoming an increasingly multifunctional living space [11]. Thereby, it serves as a good starting point for evaluating the potential of sharing as a future form of sustainable living.

\section{Research Approach and Methodology}

\subsection{Research Aim}

This paper aims to explore the potential of shared kitchens as a future sustainable living environment by studying user experiences from a Living Lab setting. The following research questions are posed: How should a shared kitchen be designed in order to optimise the conditions for both sustainability and wellbeing? How should Living Labs proceed in experimenting with shared spaces as a future form of sustainable living?

\subsection{Research Context}

The study was performed in a Living Lab in Sweden, which besides from being a research environment for future sustainable living is also a residential building containing 29 dwellings for students and researchers. Five of these are private apartments and the remaining 24 are private rooms organised into four architecturally identical 'clusters'. In each cluster, six private rooms surround a spacious common area containing a shared kitchen, two bathrooms, a living room section, a hallway and a balcony. The private rooms measure only $3.6 \times 3.6 \times 3.6 \mathrm{~m}$ and contain a sleeping loft, a small bathroom without a shower and a small 'kitchenette' without cooking possibilities.

The primary interest of this study is the shared cluster kitchen, which consists of two rows of kitchens mirrored to each other, together forming a long island placed centrally in the common area. The furnishings and appliances of the two kitchen rows are identical, apart from one base cabinet which has been replaced with a dishwasher on one side of the kitchen. Furthermore, the kitchen contains two fridge/freezer columns with a small top cabinet, two ovens with microwave function and cabinets above, two induction cooktops covered by a large hood, base cabinets with drawers and two sink units. The dining area is an extension of the countertop, which forms a bar table for up to six people at the farthest end of the kitchen, facing a large window with the balcony outside.

\subsection{Interviews}

Seven Living Lab residents signed up for an interview, representing three of the four clusters and one of the private apartments. For this paper, only the six interviews with cluster residents have been analysed. All interviews were held in the homes of the interviewees and were audio recorded, with permission from the participants. 
The interviews were of a semi structured character, following a prepared template but adding follow-up questions whenever needed. First, participants were given floor plans of the cluster living space and encouraged to talk about how they used it, mark out areas that they felt positive or negative about and describe any changes they would like to make. The focus was then turned more specifically to the kitchen area with questions regarding its use, layout, design, placement, appliances, storage possibilities, support for sustainable behaviour and sharing aspects. The final part of the interviews had a forward-looking perspective on future dwellings, sharing and sustainability.

Interview recordings were transcribed and imported to the software NVivo, where it was coded into different themes. Quotes included in the paper have been translated from Swedish to English by the first author.

\section{Findings}

\subsection{Accessing}

Several interviewees had previous experiences from shared kitchens, which were often placed at the bottom of a corridor. This kind of 'corridor kitchen' was described as a rather anonymous area where "you never know if anyone stole your food or not". One interviewee described her previous corridor kitchen as "quite disgusting since it hadn't been cleaned properly for... well a very long time”. In contrast, the central position of the cluster kitchen seemed to promote responsibility among the cluster residents due to its visual and physical presence:

"Since all doors open to the kitchen it is easy to, if someone does not clean the dishes, you can say "hey, deal with this!", while if it had been a kitchen in some corridor you don't really know... so as a shared kitchen it works very well."

It was also found that a central location may, on the other hand, result in a lack of calm spaces in the kitchen. The many doors accessing the common area limit the possibilities for utilising the space properly and one interviewee explained that:

"...what makes this apartment difficult is that you have compromises all the time [...] It becomes a hallway and a living room, it becomes a hallway and a kitchen."

\subsection{Cooking}

Although common meals were found to be a rare occurrence in the clusters, several interviewees appreciated the possibility to cook at the same time without necessarily sharing the same dish:

"Since everyone has different needs and lunch boxes, it would be such a big project to try to cook together. But at the same time, it's very nice to cook and eat together even though you somehow make your own thing."

The cluster kitchen was described as having enough space for three or four persons to cook at the same time, even though a large share of the workspace was barely used, due to a high fridge/freezer column "cutting it off" from the rest of the workspace. With the two sides of the kitchen mirrored to each other, cluster residents were able to cook 
face-to-face with another roommate instead of just facing a wall. One interviewee explained how this had made cooking and daily chores such as washing dishes more fun, which in his case had contributed to a habit of cooking at home more often.

The uneven distribution caused by having one dishwasher only resulted in a disrupted workflow. The cluster residents constantly had to walk around the long kitchen island since all dishes ended up on that side, the reason why one of the interviewees mentioned that "the kitchen doesn't work at all, it's a roundabout".

Cooking in the shared cluster kitchen was found to place high demands on the quality and performance of appliances due to frequent use and wear. In particular, two of the appliances were given several complaints. The first one was the hood:

"The hood doesn't work, it cannot be turned off or on. It's sometimes on and sometimes off and it doesn't suck out any air at all so it gets very, very smelly, so we usually open the door to the balcony, which is not so energy efficient."

The second one was the combined oven and microwave, which was explained to function poorly both as an oven and as a microwave, although one interviewee appreciated the space efficiency provided by its multifunctionality. In one of the clusters, both ovens had broken so that one did only function as an oven and the other one only as a microwave. An interviewee from that cluster thought that:

"The oven is a bad solution, you should not mix the microwave with the oven, it just gets really messy because it boils over in the microwave and then burns in the oven."

\subsection{Living and Socialising}

The possibilities for socialising was frequently mentioned as the very best aspect of the shared cluster kitchen, which was described as the centre of the cluster and the place where you most frequently meet your roommates. The dining area, located at the end of the kitchen island where it faces a large window with the balcony outside, seemed to create a pleasant environment where the residents, apart from eating, sometimes also sit down and have a chat, surf on their computers or study. However, taking the form of a bar table fixed to the cooking area, the interviewees found potential for improvements both in terms of comfort and flexibility. Several mentioned that they wanted a "real" kitchen table of normal height, where they would have the possibility to sit down and with more than six people at the same time.

\subsection{Storing}

Separation and clarity was found to be a key issue when it comes to storage of food, waste and kitchenware. Most importantly, as one of the interviewees stated, there needs to be enough storage space. For kitchenware and pantry goods, there seemed to be more than enough storage provided by the many base cabinets with drawers. Cooling and freezer space was, however, explained to be less abundant in the shared cluster kitchen. One interviewee explained that as a student, it would be good from an economic point of view to be able to store more food in the fridge and freezer.

Since the participants mainly cooked their own food, they did not share groceries to a large extent, apart from some spices, cooking oil, sauces and baking goods. The main 
part of their groceries was kept in separate drawers and on separate shelves in the fridge. For kitchenware, it was perceived as a positive thing to be able to share equipment within the kitchen but also noted that things wear out faster, causing a resistance to share items you care for with others.

Regarding waste storage, one interviewee explained that since everyone cook their own food, the cluster kitchen is subject to a large amount of waste:

"In a family you cook food together but here we cook three separate meals on each side per day, which means that we normally produce more waste."

The storage space originally dedicated to waste in the cluster kitchen was restricted to one drawer containing three separate bins in each sink cabinet, comparable to the configuration of a standard Swedish kitchen. It became clear that this storage space was inadequate, both in terms of the amount and the opportunity to separate fractions. Waste separation was consequently described as a challenge by all interviewees. In one cluster, waste storage was expanded to other kitchen drawers as well and in another cluster, waste ended up in the hallway once the standard drawer was filled up. Among the cluster residents, waste sorting was explained to be performed to varying degree and it was a subject that occasionally caused some irritation. Some of them had stopped sorting some categories of waste since they moved there just because it was too big of a project:

"Normally, I would have sorted soft plastics and everything but there is no space so I can't take it."

\subsection{Cleaning}

In the Living Lab, there is a cleaning service taking care of the bathrooms and floors in the common area of the clusters, which mainly leaves the task of cleaning up the kitchen to the residents. In the three clusters included in the study, cleaning was not organised by the residents according to any system. In order for it to work, one interviewee explained that:

"I think the most important thing is that everyone has a similar sense of responsibility towards the common areas, because if everyone would accept that it's messy, or could live with having it that way, then it would be okay, but as long as someone deviates from it - that's when it turns into a problem."

The fact that you need to take the responsibility to clean up after yourself was mainly seen a positive thing among the interviewees, even though it may demand a little more effort compared to a single household kitchen where leaving the mess for a while longer doesn't affect anyone else. However, as one of the interviewees said: "you can't expect it to be pedantic in a shared kitchen". 


\section{Discussion and Conclusions}

A central position of the kitchen in relation to other (private) living spaces was found to support socialising but also to promote a sense of responsibility in its usage. However, the kitchen also needs to include calm spaces to support wellbeing. Regarding cooking, it can be concluded from this study that a shared kitchen requires appliances of high quality and a good amount of workspace supporting several people to cook at the same time. When designing a shared kitchen, there is an opportunity to focus on the social aspects of cooking and bring people together rather than keeping them apart at the kitchen's different ends.

Storage space need to be spacious enough with clearly delimitated sections, especially in order to support a well-functioning system for waste recycling. Having a common sense of responsibility among all users was regarded as key for shared products as well as shared tasks such as handling waste, washing dishes and cleaning up.

A suggestion for future research in Living Labs is to investigate requirements placed on shared kitchens used by a wider variety of households. Topics that need further consideration are how to achieve higher flexibility and adaptability for different use cases and how to improve the balance between centrality and calm spaces in the kitchen.

Acknowledgments. This work is part of the European collaboration project The Circular Kitchen, supported by the Climate-KIC initiative of the EIT, Area of Focus Urban Transition.

\section{References}

1. Eurostat: Household composition statistics. https://ec.europa.eu/eurostat/statistics-explained/ index.php/Household_composition_statistics

2. Keilman, N.: Biodiversity: the threat of small households. Nature 421(6922), 489-490 (2003)

3. Klocker, N., Gibson, C., Borger, E.: Living together but apart: material geographies of everyday sustainability in extended family households. Environ. Plann. A. 44(9), 2240-2259 (2012)

4. Gram-Hanssen, K.: Efficient technologies or user behaviour, which is the more important when reducing households' energy consumption? Energ. Effi. 6(3), 447-457 (2013)

5. Jackson, T.: Where is the "wellbeing dividend"? nature, structure and consumption inequalities. Local Environ. 13(8), 703-723 (2008)

6. Belk, R.: Sharing. J. Consum. Res. 36(5), 715-734 (2010)

7. Leismann, K., Schmitt, M., Rohn, H., Baedeker, C.: Collaborative consumption: towards a resource-saving consumption culture. Resources 2(3), 184-203 (2013)

8. Jarvis, H.: Saving space, sharing time: integrated infrastructures of daily life in cohousing. Environ. Plan. A. 43(3), 560-577 (2011)

9. Mont, O.: Institutionalisation of sustainable consumption patterns based on shared use. Ecol. Econ. 50(1-2), 135-153 (2004)

10. Hagbert, P.: "It's just a matter of adjustment": residents' perceptions and the potential for low-impact home practices. Hous. Theory Soc. 33(3), 288-304 (2016)

11. Hand, M., Shove, E., Southerton, D.: Home extensions in the United Kingdom: space, time, and practice. Environ. Plan. D: Soc. Space 25(4), 668-681 (2007) 